\title{
The trefoil gene family are coordinately expressed immediate-early genes: EGF receptor- and MAP kinase-dependent interregulation
}

\author{
Douglas Taupin, Deng-Chyang Wu, Woo-Kyu Jeon, Kathryn Devaney, \\ Timothy C. Wang, and Daniel K. Podolsky
}

Gastrointestinal Unit and Center for the Study of Inflammatory Bowel Disease, Massachusetts General Hospital and Harvard Medical School, Boston, Massachusetts 02114, USA

Address correspondence to: Daniel K. Podolsky, Massachusetts General Hospital, Gastrointestinal Unit, 55 Fruit Street, GRJ-719, Boston, Massachusetts 02114, USA. Phone: (617) 726-7411; Fax: (617) 724-2136; E-mail: Podolsky.Daniel@mgh.harvard.edu

Douglas Taupin's present address is: Signal Transduction Unit, Peter MacCallum Cancer Institute, St. Andrew's Place, East Melbourne 3002, Victoria, Australia.

Deng-Chyang Wu's present address is: Gastrointestinal Unit, Kaohsiung Medical College Hospital, Taipei, Taiwan.

Woo-Kyu Jeon's present address is: Gastrointestinal Unit, Department of Internal Medicine, Kangbuk Samsung Hospital, 108 Pyung-Domg, Jongno-Ku, Seoul 100-634, Korea.

Received for publication March 5, 1998, and accepted in revised form March 19, 1999.

The trefoil gene family of mucus cell-secreted proteins is a critical mediator of gastrointestinal mucosal restitution. Transcription of trefoil genes is induced during mucosal repair, but the regulatory mechanisms involved are unknown. Mice deficient in the intestine-specific peptide intestinal trefoil factor (ITF), in which colonic restitution is lethally impaired, showed reduced expression of the gastric trefoil genes $S P$ and $p S 2$, suggesting that trefoil peptides may individually regulate transcription of the entire family. In gastric cell lines, the trefoils were shown to act in a manner suggestive of immediate-early genes capable of auto- and cross-induction through cis-acting regulatory regions. Trefoil-mediated transcriptional regulation required activation of the Ras/MEK/MAP kinase signal transduction pathway. EGF receptor (EGF-R) activation was also necessary for trefoil auto- and cross-induction, and both spasmolytic polypeptide (SP) and ITF stimulation of gastric cell lines led to phosphorylation of EGF-R. Nevertheless, ITF and ITF-thioredoxin cell surface binding at $4{ }^{\circ} \mathrm{C}$ colocalized not with EGF-R, but with CD71, which is found in clathrin-coated pits, suggesting that integration of trefoil peptide responses may occur after internalization. As EGF-R expression is itself strongly induced after mucosal damage, the trefoil/EGF-R relationship may be pivotal in the generation and maintenance of the mucosal repair phenotype.

J. Clin. Invest. 103:R31-R38 (1999).

\section{Introduction}

An essential feature of mucosal surfaces is the ability of epithelial cells to spread and migrate across the basement membrane to cover shallow defects. This rapid process, which occurs independently of cell proliferation over the course of hours, is termed restitution (1-3). Though a number of cytokines and growth factors, including EGF, TGF- $\alpha$ and $-\beta$, and HGF, have been shown to initiate migration of gastrointestinal cells in vitro (4-8), a critical role in restitution is played by peptides of the trefoil gene family, comprising the intestinal peptide intestinal trefoil factor (ITF) and the gastric peptides SP erosions caused by these agents $(12,13)$. Mice made pS2 deficient develop hyperproliferative nodules and adenomas in the gastric antrum, the result either of cumulative injury or loss of an undefined antiproliferative action of this peptide (14). Whether these actions of trefoil peptides occur through binding to specific receptors has not been established.

Under normal circumstances, the trefoil genes are expressed in a cell-specific manner. SP is a product of the gastric mucous neck cell $(15,16)$, with pS2 expressed by surface (foveolar) mucous cells of the stomach $(17,18)$. ITF is a major peptide product of the intestinal goblet cell, with less abundant expression in gastric glands $(16,19,20)$. All 3 genes are rapidly induced after mucosal injury, essential qualities for proteins active in mucosal repair. This induction is not tissue specific: ITF may be strongly induced in the stomach (21), while $\mathrm{SP} / \mathrm{pS} 2$ may be strongly induced in the intestine $(22,23)$. There is sustained induction of these genes in association with persistence of the repair phenotype and with true metaplasia, including gastric metaplasia of the duodenum and pseudopyloric metaplasia of the intestine (22-24). The mechanisms regulating expression of these genes after injury are poorly understood, but coordination of their transcription seems likely. We show here that ITF-deficient mice have reduced SP and pS2 gene expression and define in model cell lines the transcriptional and signaling events responsible for this reduced expression. 


\section{Methods}

Plasmids and peptides. The 1,100-bp human pS2 promoter was cloned from a human genomic library (PromoterFinder; CLONTECH Laboratories Inc., Palo Alto, California, USA) by PCR using nested proprietary upstream and gene-specific downstream primers (primer 1: 5'-AGCGTGCCGAGGGCCAGCAT-3'; primer 2: 5'-CGAGATCTCCTCTCTGCTCCAAAG-3') and subcloned into the MluI-BglII sites of the luciferase reporter vector pGL2 (Promega Corp., Madison, Wisconsin, USA). A genomic clone encompassing hSP was isolated from the human genomic library EMBL3 SP7/T6 (CLONTECH Laboratories Inc.) using standard screening protocols. Positive clones were identified by hybridization with a $\left[\alpha-{ }^{32} \mathrm{P}\right] \mathrm{dCTP}-$ labeled random-primed rat SP cDNA probe (15) and were plaque purified. Phage DNA incorporating a 23-kb Xba1 insert was harvested by liquid culture lysis (25). Presence of promoter sequence was confirmed by Southern blotting using an end-labeled oligonucleotide probe (5'-GAGCTGGGCGTCTGGAGGACGAGGGTCTGGAGGAACAGGgGCCTGGAGGATG-3'). This insert was then subjected to PCR to amplify the 820-bp promoter fragment upstream of the initiating ATG (5'-GGGGTACCACACTTTTCCCTCTT-3', 5'-GAGGTACCCTGTCAGCCTCATCT-3'; downstream primer: 5'-CGAGATCTAGCTCAGCTGCACCCC-3'), which was subcloned into the KpnI-BglII sites of pGL2. Both pS2 and SP promoters were confirmed by DNA sequencing and comparison with the database; in particular, presence of the "estrogen response element" (26) of pS2, 444 bp upstream of the ATG, was confirmed.

The vector PMT2T-PAC1, expressing a constitutively active ERK-specific phosphatase PAC1 (27), was a gift from K. Kelly (National Cancer Institute, Bethesda, Maryland, USA). The Ha-ras-Asn-17 (RasN17) construct expressing a dominant-negative Ras that preferentially binds GDP over GTP (28) was a gift from G.M. Cooper (Harvard Medical School). The kinase-deficient EGF receptor (EGF-R) expression plasmid pRK5HER653 (29) was a gift from M. Korc (University of California-Irvine, Irvine, California, USA). The human gastrin promoter fused to luciferase, containing Ras and EGF response elements (30), was a gift from D. Chung (Massachusetts General Hospital/ Harvard Medical School). The Renilla luciferase reporter vector pRL-TK was obtained from Promega Corp. Purified recombinant human SP and ITF, produced in yeast, have been described previously $(9,31)$.

Cell culture. The human gastric cancer cell lines AGS and KATO-III and the human colon cancer cell lines HT-29 and LS-174 were obtained from the American Type Culture Collection (Rockville, Maryland, USA). AGS and HT-29 cells were grown in DMEM supplemented with $10 \%$ FCS, KATO-III cells in Iscove's medium with $20 \%$ FCS, and LS174 cells in MEM with 10\% FCS; all media were supplemented with $4 \mathrm{mM}$ L-glutamine, $50 \mathrm{U} / \mathrm{mL}$ penicillin, and $50 \mu \mathrm{g} / \mathrm{mL}$ streptomycin. Cells were grown in $5 \% \mathrm{CO}_{2}$ at $37^{\circ} \mathrm{C}$. Northern blotting and immunoblotting for trefoil peptides. ITF null mice have been described previously (11). Mouse gastric RNA preparation and Northern blotting were also performed as described previously (11). For in vitro studies, RNA was extracted from confluent, serum-starved cells in 6well, 3.5-cm-diameter plates, using Trizol (GIBCO BRL, Grand Island, New York, USA) according to the manufacturer's instructions. Five micrograms total RNA was loaded onto denaturing $1 \%$ agaroseformaldehyde gels, and Northern blotting was performed as described (11). The murine SP and pS2 and human ITF probes have been described $(11,15,20)$. Human SP (252 bp) and pS2 (387 bp) cDNA probes were amplified from oligo-dT-primed human gastric RNA by reverse transcription and PCR (GeneAmp; Perkin-Elmer, Norwalk, Connecticut, USA) performed according to the manufacturer's instructions. The SP product was cloned into the TA cloning vector PCR 2.1 (Invitrogen, San Diego California, USA), and a 317-bp KpnI-SacI pS2 fragment was cloned into the corresponding sites of pBluescript (Stratagene, La Jolla, California, USA). Primers were as follows: hSP 5 'AGCCCCCATAACAGGACGAACTG-3' and 5'-GCACCAGGGCACTTCAAAGATGA-3'; hpS2 5'-GGGGTCGCCTTTGGAGCAGA-3' and $5^{\prime}$-CGTGTCTGAGGTGTCCGGTG-3'. Immunoblotting of mouse gastric tissue extracts was performed as described previously (11), using antisera directed against rat SP (15) or mouse pS2 (a gift from P. Chambon, INSERM, Strasbourg, France).

Transient transfection assays. KATO-III and AGS cells grown in $3.5-\mathrm{cm}$ wells were transiently transfected by calcium phosphate coprecipitation essentially as previously described (32), with $1 \mu \mathrm{g}$ pS2-luc, hSP-luc, and PMT2T-PAC1, and $2 \mu \mathrm{g}$ N17Ras or pRK5-HER653. For cotransfections with pRK5-HER653 and treatment with EGF, and for treatment with PD098059, transfection efficiency was monitored by cotransfection with $1 \mu \mathrm{g}$ pRL-TK (Promega Corp.) under control of the TK promoter, which lacks SV40 early enhancer elements. Twelve hours after transfection, cells were rinsed with PBS and then changed to serum-free medium (Ultraculture; BioWhittaker, Walkersville, Maryland, USA) before stimulation with recombinant peptides at the indicated final concentrations or $10 \mu \mathrm{M}$ of the MEKkinase inhibitor PD098059 (New England Biolabs, Beverly, Massachusetts, USA). Cells were lysed and assayed 24 hours following stimulation using a commercial firefly or Renilla luciferase assay system (Promega) and luminometer (Monolite 2010; Analytical Luminescence Laboratory, San Diego, California, USA). Results shown are representative of 3 similar experiments per- formed in triplicate and are expressed as mean \pm SE of relative luciferase units as a percentage of RSV luciferase, or where indicated as relative luciferase units corrected for transfection efficiency.

Immunoprecipitation and immunoblotting. Cells were incubated in serum-free media (AGS cells) or in $0.2 \%$ serum (KATO-III) for at least 12 hours before stimulation with trefoil peptides, $50 \mathrm{nM}$ human EGF (R\&D Systems Inc., Minneapolis, Minnesota, USA) or $50 \mu \mathrm{M}$ genistein (Sigma Chemical Co., St. Louis, Missouri, USA), rinsed in cold PBS, and then lysed on ice in lysis buffer $(0.5 \%$ NP-40, $10 \mathrm{mM}$ Tris-HCL [pH 7.4], $150 \mathrm{mM}$ $\mathrm{NaCl}, 1 \mathrm{mM}$ EDTA, $10 \mu \mathrm{g} / \mathrm{mL}$ aprotinin, $100 \mathrm{mM}$ phenylmethylsulfonyl fluoride, 10 $\mu \mathrm{g} / \mathrm{mL}$ leupeptin) containing $500 \mu \mathrm{M}$ sodium orthovanadate, $100 \mathrm{mM}$ sodium fluoride, and $10 \mathrm{mM}$ sodium pyrophosphate as phosphatase inhibitors. Lysates were centrifuged $\left(12,000 \mathrm{~g}, 15\right.$ minutes, $\left.4^{\circ} \mathrm{C}\right)$, and $500 \mu \mathrm{g}$ protein from the resulting supernatants was clarified with rabbit $\operatorname{Ig}$ (R\&D Systems Inc.) or protein A-Sepharose (Pharmacia Biotech Inc., Piscataway, New Jersey, USA), incubated overnight with 1-3 $\mu \mathrm{g}$ of antibodies, and then precipitated with protein ASepharose. Antibodies were mouse monoclonal anti-ERK1 or anti-ERK2, anti-c-Jun (all from Transduction Laboratories, Lexington, Kentucky, USA), or sheep anti-EGF-R (Upstate Biotechnology Inc., Lake Placid, New York, USA). Immunoprecipitates were washed 3 times in lysis buffer, SDS sample buffer was added, and samples were boiled and then subjected to SDS-PAGE $(7.5 \%$ gel) followed by immunoblotting with anti-phosphotyrosine antibodies (4G10; Upstate Biotechnology Inc.) and enhanced chemiluminescence (Renaissance; Du Pont NEN Research Products, Boston Massachusetts, USA).

Immune complex kinase assays. Immunoprecipitates were washed twice with lysis buffer and once with kinase reaction buffer $(40 \mathrm{mM}$ HEPES [pH 7.5], $10 \mathrm{mM} \mathrm{MgCl}_{2}, 3 \mathrm{mM}$ $\mathrm{MnCl}_{2}$ ), resuspended in $50 \mu \mathrm{L}$ kinase buffer containing $7.5 \mu \mathrm{g}$ myelin basic protein or 0.3 $\mu \mathrm{g} \mathrm{c}$-Jun (Sigma Chemical Co.) and $10 \mu \mathrm{Ci}[\alpha-$ $\left.{ }^{32} \mathrm{P}\right] \mathrm{dATP}$, and then incubated for 30 minutes at $25^{\circ} \mathrm{C}$. Reactions were stopped by heating the samples to $90^{\circ} \mathrm{C}$ in SDS sample buffer and were analyzed by $11.5 \%$ SDS-PAGE and autoradiography.

Immunocytochemistry. AGS or HT-29 cells were grown on glass slides, then changed to serum-free conditions for 8 hours and cooled to $4^{\circ} \mathrm{C}$. ITF or thioredoxin-coupled ITF (Taupin, D., et al., manuscript in preparation) was added at $10 \mu \mathrm{g} / \mathrm{mL}$ for $30 \mathrm{~min}$ utes; then cells were washed with PBS and fixed in $2 \%$ paraformaldehyde for 30 minutes. After blocking with normal goat or horse serum, paired antibodies were added as follows: monoclonal anti-thioredoxin (Invitrogen) with rabbit anti-EGF-R (Santa Cruz Biotechnology Inc., Santa Cruz, California, USA); or anti-ITF (20) with monoclonal 

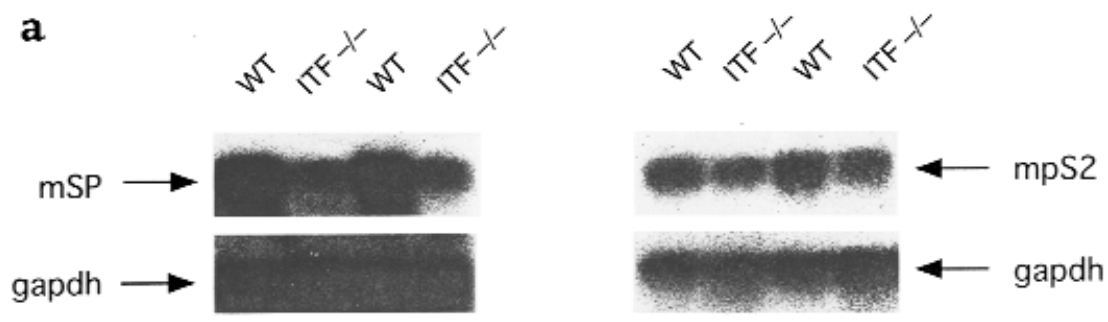

b

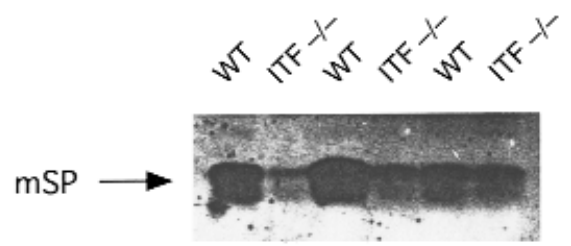

Figure 1

Gastric trefoil peptide RNA and protein is reduced in $1 \mathrm{TF}^{-/-}$compared with wild-type (Sv129/C57BI/6) mice. (a) Northern blots of mouse gastric antrum. Total RNA was hybridized with an $\left[\alpha-{ }^{32} \mathrm{P}\right] \mathrm{dCT}$-labeled $\mathrm{rSP}$ cDNA probe (15), mouse ITF cDNA probe (51), mouse-specific $\mathrm{pS} 2$ probe (11), or a probe for the constitutive GAPDH transcript as described in Methods. (b) Western blot of gastric mucosal lysates resolved by SDS-PAGE, transferred to polyvinylidene difluoride membrane, incubated with rSP antibody (15), and viewed by enhanced chemiluminescence.
anti-EGF-R (Transduction Laboratories) or biotinylated anti-CD71 (PharMingen, San Diego, California, USA). Secondary antibodies used were horse anti-mouse FITC, donkey anti-rabbit FITC, or streptavidin/Texas Red (all from Vector Laboratories, Burlingame, California, USA) or cy-3 donkey anti-mouse (Jackson ImmunoResearch Laboratories Inc., West Grove, Pennsylvania, USA). Slides were mounted with glycerol/PBS and coverslipped. A confocal scanning dual-laser microscope (Model 600; Bio-Rad Laboratories Inc., Hercules, California, USA) equipped with a Nikon Diaphot 200 (Nikon Inc., Melville, New York, USA) was then used to Double-label images were collected sequentially and were of similar intensity.

\section{Results}

$S P$ and $P S 2$ expression is reduced in ITF null mice. ITF is predominantly expressed by the intestinal goblet cell, with low levels of expression in the stomach $(16,19$, 20). Unexpectedly, when expression of other trefoil peptides was assessed in $\mathrm{ITF}^{-/-}$mice, gastric expression of SP was found to be consistently reduced ( $38 \pm 9$ $\%$ of control; $n=6$ ) (Figure 1a), compared with wild-type mice (Sv129 backcrossed into C57Bl/6J). The pS2 mRNA was similarly reduced in $\mathrm{ITF}^{-/-}$animals compared with controls $(56 \pm 12 \%$ of control; $n=6$ ) (Figure 1a). SP (Figure 1b) and pS2 (data not shown) peptide content were also reduced. (by $40 \%$ and $65 \%$, respectively). Diminished SP expression has been noted in mice lacking pS2 (14). ITF and SP show auto- and cross-induction obtain multiple $0.25-\mu \mathrm{m}$ optical sections. recombinant ITF or SP at $10-100 \mu \mathrm{g} / \mathrm{mL}$ (approximately 1-10 $\mu \mathrm{M}$, within the physiologic range). Stimulation of cell lines with either ITF or SP yielded increased steady-state hSP mRNA within 10 minutes, with maximal stimulation seen after 30 minutes (Figure 2, top lane). ITF gene expression was also induced by SP stimulation (Figure 2, third lane). Similarly, ITF increased ITF expression in cells of gastric origin (2.4fold at 10 minutes, 3.0 -fold at $30 \mathrm{~min}$ utes, and 1.6-fold at 60 minutes). Autostimulation was also seen in the intestinal cell line LS174 (data not shown). Although low in the cell lines studied, pS2 expression was also induced by both ITF and SP compared with unstimulated cells (Figure 2, third lane). Overall SP led to 3.2-, 2.4-, and 1.3fold stimulation of SP, ITF, and p52, respectively, while ITF treatment resulted in 4.2-, 3.0-. and 2.3-fold stimulation of SP, ITF, and p52, respectively (Table 1). Pretreatment with cycloheximide $(25$ $\mu \mathrm{g} / \mathrm{mL}, 30$ minutes before stimulation) enhanced trefoil peptide stimulation of pS2 and SP mRNA (Figure 2, center lane). These features are suggestive that these act as immediate-early response genes (35).

Regulation of gastric trefoil expression by EGF-R ligands has been suggested $(23,36)$, and pS2 transcription is markedly enhanced by EGF in the breast cancer cell line MCF-7 (26). The effect of EGF stimulation on trefoil Indeed, in cells of gastric origin, EGF stimulated increased trefoil expression (Table 1 and Figure 2). gene expression was therefore assessed.

Table 1

Cross-induction of trefoil peptide-encoding mRNA

\begin{tabular}{lccc}
\hline & SP & ITF & PS2 \\
Control & 1 & 1 & 1 \\
SP 10 minutes & $2.84 \pm 0.21$ & $1.99 \pm 0.30$ & $1.22 \pm 0.13$ \\
ITF 10 minutes & $3.23 \pm 0.33$ & $2.42 \pm 0.26$ & $1.31 \pm 0.19$ \\
SP 30 minutes & $3.98 \pm 0.42$ & $3.07 \pm 0.41$ & $1.84 \pm 0.20$ \\
ITF 30 minutes & $4.2 \pm 0.39$ & $3.01 \pm 0.34$ & $2.31 \pm 0.24$ \\
SP 60 minutes & $6.07 \pm 0.83$ & $2.63 \pm 0.27$ & \\
ITF 60 minutes & $4.39 \pm 0.56$ & $1.56 \pm 0.18$ & $1.99 \pm 0.23$ \\
SP 30 minutes CHX & $5.27 \pm 0.48$ & $3.98 \pm 0.39$ & $2.44 \pm 0.21$ \\
ITF 30 minutes CHX & $6.36 \pm 0.75$ & $3.52 \pm 0.51$ & $2.18 \pm 0.16$ \\
& & & $1.92 \pm 0.17$ \\
EGF & $5.61 \pm 0.64$ & $3.12 \pm 0.25$ &
\end{tabular}

Confluent KATO-III cells were serum starved for 6 hours. ITF $=100 \mathrm{ng} / \mu \mathrm{L} ; \mathrm{EGF}=100 \mathrm{ng} / \mu \mathrm{L}$. Values represent mean $\pm \mathrm{SE}$ density normalized to GAPDH for each probe relative to control for 6 determinants. CHX, cycloheximide. 


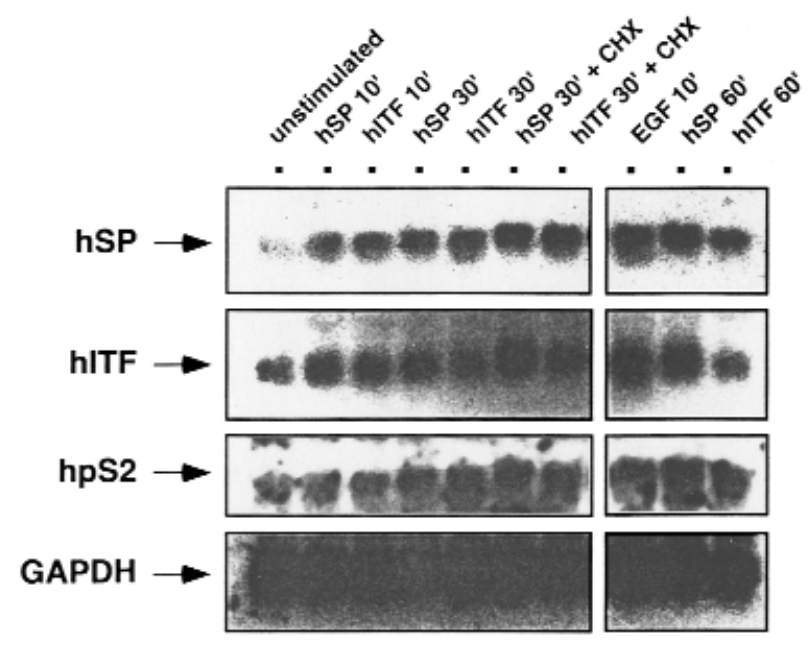

Figure 2

Expression of trefoil peptide-encoding mRNA in gastric cells after stimulation with trefoil peptides. KATO-III cells were grown to confluence, serum-deprived ( $0.5 \%$ serum), and then stimulated for the indicated times with recombinant ITF $(100 \mu \mathrm{g} / \mathrm{mL}), \mathrm{SP}(100 \mu \mathrm{g} / \mathrm{mL})$, or EGF $(100 \mathrm{ng} / \mathrm{mL})$ in the absence or presence of cycloheximide $(25 \mu \mathrm{g} / \mathrm{mL})$. Northern blots were performed with $[\alpha$ ${ }^{32} \mathrm{P}$ ]dCTP-labeled cDNA probes as described in Methods. CHX, cycloheximide.

$I T F$ and SP activate cis-regulatory elements in SP and $p S 2$ promoters. To assess the specificity and define the mechanism of the transcriptional response to extracellular trefoils, hSP and hpS2 promoters (Figure 3a) were cloned into luciferase reporter vectors, and these constructs were transfected into KATOIII and AGS cells. After transfection, the cells were stimulated with a single dose of SP or ITF after 24 hours, and luciferase activity was measured at 48 hours. In both AGS (Figure 3b) and KATO-III (data not shown) cells, ITF treatment stimulated luciferase activity driven by either the hSP promoter (Figure $3 \mathrm{~b}$, bottom left) or the hpS2 promoter (Figure $3 \mathrm{~b}$, bottom right), with maximum effect seen at $10 \mu \mathrm{g} / \mathrm{mL}$ ITF (approximately $1.5 \mu \mathrm{M}$ ). SP treatment also stimulated hSP (Figure 3a, top left) and hpS2 (Figure 3b, top right) promoters, with peak effect at $10 \mu \mathrm{g} / \mathrm{mL}$ $(0.8 \mu \mathrm{M})$ and $100 \mu \mathrm{g} / \mathrm{mL}$, respectively.
No stimulation of a control vector (pGL2-luciferase) was seen.

Cross-regulation by trefoil peptides is MAP kinase dependent. We next defined the intracellular signaling molecules that mediate the transcriptional response to trefoils. MAP kinases were evaluated as candidates for this role because their activation may be necessary for epithelial cell motility and because they mediate transcriptional responses of other immediate-early genes active in gastrointestinal epithelium $(37,38)$. MAP kinase activation was assessed by in vitro kinase assays after stimulation of KATOIII (Figure 4a) or AGS cells with ITF or SP (data not shown). Normalized to ERK expression (by reprobing with antiERK) stimulation was $14.5 \pm 2.3$-fold within 1 minute after addition of ITF ( $n$ $=4)$. Maximum activation occurred 5 minutes after stimulation and was blocked by pretreatment with the protein kinase inhibitor genistein (Figure $4 a$, lane $5 G$ ). No activation of Jun kinase (Figure 4b) or p38 kinase (data not shown) occurred at the concentrations used. This MAP kinase activation was necessary for cross-induction of trefoil gene transcription, as cotransfection of hpS2 (Figure 4c) and hSP (data not shown) promoters with an expression construct for the phosphatase PAC1, which inactivates ERK1 and ERK2 after nuclear translocation (27), prevented ITF-induced transcriptional activation. Pretreatment of transfected cells with a
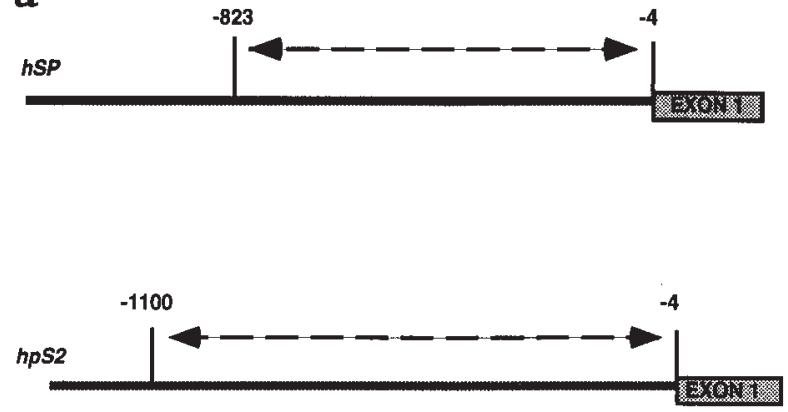

\section{Figure 3}

Effect of recombinant trefoil peptides on expression of human pS2 and SP reporters in transient transfection. (a) hSP (820 bp) and hpS2 (1,097 bp) promoters were cloned as detailed in Methods and subcloned into the luciferase reporter vector pGL2. (b) AGS cells were transfected with hSP-luc (left panels) or hpS2-luc (right panels) reporter constructs, followed by stimulation with human SP (top panels) or human ITF (bottom panels) peptides at the indicated final concentrations for 24 hours, as described in Methods. Results shown (mean \pm SE) are representative of 3 independent experiments performed in triplicate. b b hSP PROMOTER pS2 PROMOTER
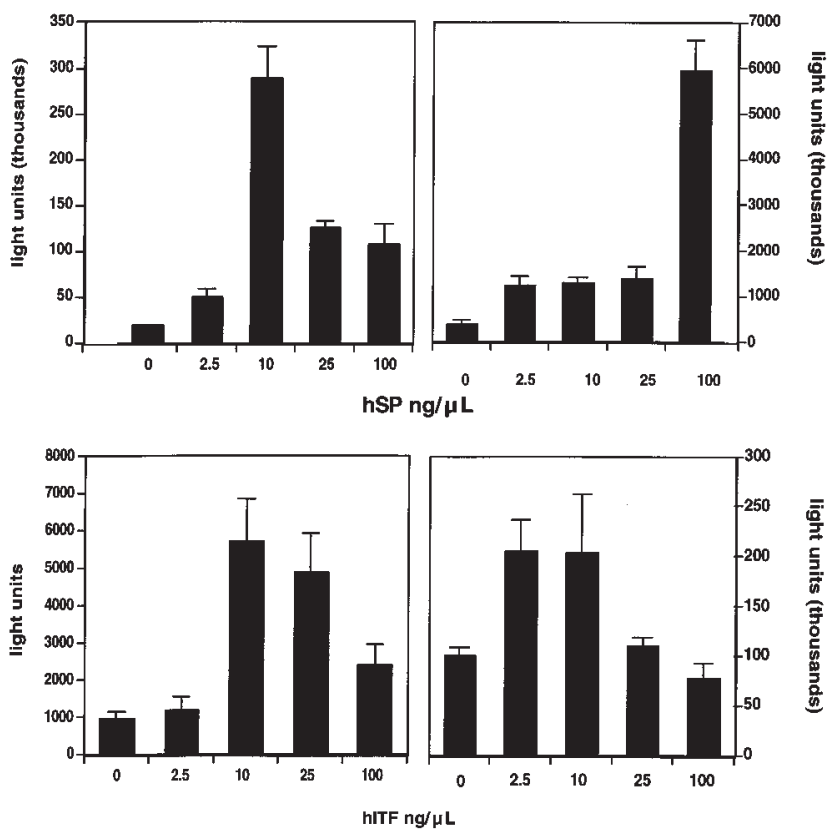
a
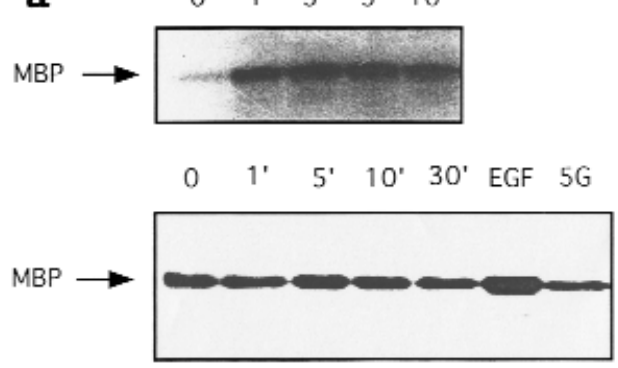

b

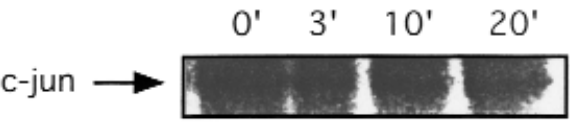

c

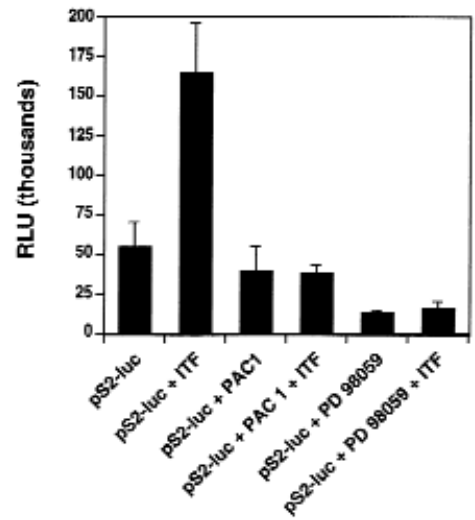

Figure 4

(a) MAP kinase activation by trefoil peptides. Confluent, serum-deprived KATO-III cells (top) or serum-starved AGS cells (bottom) were stimulated for the indicated times with human ITF or SP at $10 \mathrm{ng} / \mu \mathrm{L}$, human EGF at $100 \mathrm{ng} / \mathrm{mL}$, or genistein $50 \mu \mathrm{M}$ (lane $5 \mathrm{G}$ ). Cells were harvested by in-plate lysis in the presence of protease inhibitors, and $500 \mu \mathrm{g}$ protein was immunoprecipitated with polyclonal anti-ERK1. Immunoprecipitates were incubated with myelin basic protein as substrate in the presence of $\left[\gamma^{32} \mathrm{P}\right] \mathrm{dATP}$ and resolved on SDS-PAGE.

(b) Lack of Jun kinase activation by addition of hSP to KATO-III cells. Cell lysates as above were immunoprecipitated with monoclonal anti-c-Jun. Kinase reactions were performed with c-Jun as substrate. (c) MAP kinase activation is necessary for cross-regulation of trefoil peptide transcription. AGS cells were transiently transfected with pS2-luc and/or cotransfected with an expression construct encoding the ERK-dephosphorylase PAC-1 (PMT2T-PAC1). Twelve hours after transfection, cells were stimulated with ITF (10 ng/ $\mu \mathrm{L})$ and/or the MEK-1 inhibitor PD098059 (25 $\mu \mathrm{M})$.

the MEK1 inhibitor PD098059 (Figure 4c), which blocks activation of the MAP kinase kinase MEK1 (39), also prevented pS2 (shown) and hSP (data not shown) induction by $10 \mathrm{ng} / \mu \mathrm{L}$ ITF.

Cross-regulation by trefoil peptides requires functional Ras. These observations suggested that Ras activation may be necessary to transduce the cross-regulation by trefoils, because Ras is a known upstream activator of the MAPK pathway (38). To test this assumption the dominant-negative Ras mutant RasN17 (28) was expressed in AGS cells by cotransfection with the hpS2 promoter, as shown in Figure 5. RasN17 suppressed both basal and ITF-induced hpS2-driven reporter activity. Suppression of Ras signaling by RasN17 was confirmed by the ability to block EGF-induced trans-activation of the human gastrin promoter (Figure 5), previously reported to be Ras dependent (30). Consistent with this data, cotransfection of wild-type c-Ha-Ras was able to strongly induce both basal and ITFinduced pS2 and SP reporter activity (data not shown).

Cross-regulation by trefoils requires EGF$R$ phosphorylation. Although pS2 and SP are normally expressed by the gastric surface mucous cells and mucous neck cells of the stomach $(15,17,40)$ with minimal colonic expression, and abundant ITF expression is restricted to the intestine, all 3 genes are induced in regenerative mucosa adjacent to sites of chronic ulceration throughout the gastrointestinal tract (22). This pattern of expression has also been observed in several models of experimental mucosal injury $(21,36)$. This regenerative mucosa also shows strong upregulation of the EGF-R $(41,42)$. Therefore, we stimulated AGS cells with ITF and SP and assessed EGF-R phosphorylation. Both ITF (Figure 6a) and SP (data not shown) at $10 \mu \mathrm{g} / \mathrm{mL}$ caused transient EGF-R phosphorylation, which was maximal 5 minutes after stimulation. Similar results were recently shown for ITF in the intestinal cell line HT-29 (43).

These findings suggested that activation of EGF-R might mediate cross-regulatory signals from trefoil peptides. To test this hypothesis, AGS cells were cotransfected with pS2-luciferase and the expression plasmid pHER653, encoding a kinase domain-deficient EGF-R that functions as a dominant negative (29). Interestingly, while EGF treatment leads to only a small increase in ITF, SP, and pS2 steady-state mRNA, as assessed by Northern blotting (Figure 2), pS2 promoter activity itself was highly inducible by EGF in AGS cells (Figure $6 \mathrm{~b})$, as observed in another cell line (26). Induction by EGF was partially blocked by cotransfection with pHER653. A similar degree of suppression of EGF-stimulated human gastrin promoter activity was seen (data not shown). Finally, when AGS cells were transfected with pHER653, stimulation of human pS2 promoter activity by $10 \mu \mathrm{g} / \mathrm{mL}$ ITF was negligible (Figure $6 \mathrm{~b}$ ). This reduction of luciferase activity was statistically significant $(P<0.05, t$ test $)$, indicating that the EGF-R at least partly mediates cross-regulation of $\mathrm{pS} 2$ transcription.

ITF colocalizes with clathrin-coated sites but not with the EGF-R. Since the EGF-R appears to be functionally downstream of ITF-activated pathways, we considered whether ITF itself may be an alternate ligand for the EGF-R. ITF shares with EGF and TGF- $\alpha$ the property of a compact structure resulting from 3 intrachain disulfide bonds, albeit in EGF-R ligands, cysteines 1-3, 2-4, and 5-6 form disulfide bonds, while the cysteine pairs are 1-5, 2-4, and 3-6 in the trefoil peptides (44). However, no ITF binding to the EGF$\mathrm{R}$ could be demonstrated by coprecipitation or by cross-linking experiments in the human cell lines AGS and HT29 or the rat intestinal line IEC-6 (data not shown). To determine how ITF binding related to EGF-R sites, an excess of ITF or an $\mathrm{NH}_{2}$-terminal fusion of ITF with thioredoxin were added to AGS or HT-29 cells under nonpermissive conditions, and immunocytochemistry was performed. Minimal colocalization of ITF and/or 


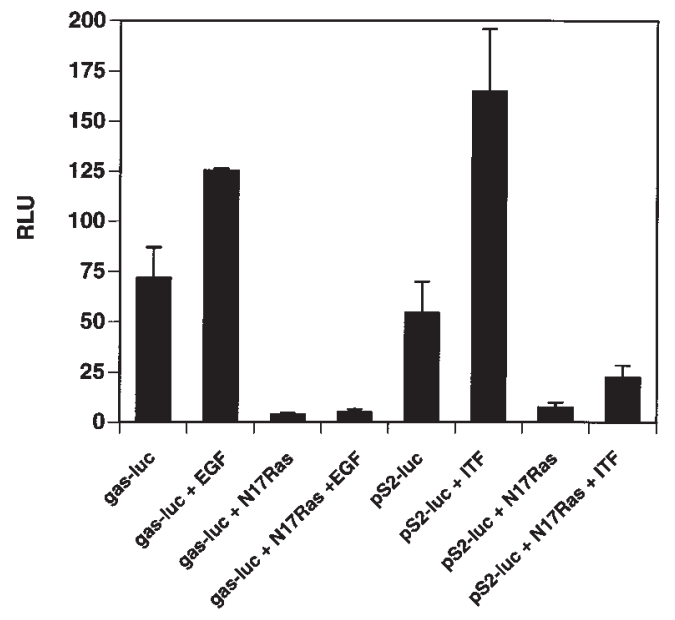

ITF-thioredoxin with the EGF-R was observed, with much of the respective signals remaining discrete (Figure 7 , top panels). Rather, the majority of ITF surface localization occurred in a pattern that suggests binding to clathrin-coated pits. This was confirmed by colocalization of ITF with the transferrin receptor CD71 (Figure 7 , bottom panels). Since the EGF-R is also recruited to clathrin-coated pits after activation (45), this implies that transactivation of the EGF-R by ITF may occur indirectly.

\section{Discussion}

The initial finding of this study was reduced SP and PS2 expression in ITF null mice. This suggested the possibili-

\section{Figure 5}

Functional Ras is required for basal and trefoil-dependent pS2 transcription. AGS cells were cotransfected with a human gastrin promoter-luciferase reporter plasmid (gas-luc) or the pS2 promoter luciferase construct with or without dominant-negative Ras (N17Ras). To confirm the effect of N17Ras, the effect of EGF (10 $\mathrm{ng} / \mathrm{mL}$ ) on Ras-dependent (30) activation was assessed.

ty of a regulatory effect on SP and pS2 transcription by ITF, which was confirmed by an increase in steady-state trefoil mRNA in gastrointestinal cell lines treated with hSP or hITF and increased SP and pS2 promoter activity in transfected gastric cell lines after stimulation. Signaling studies indicated that transcriptional activation occurs through a Ras/MEK/MAPK pathway, which also involves EGF-R phosphorylation and kinase activity.

While we have shown involvement of classic Ras/MAPK signaling pathways in gastric cell lines, whether this effect, or any function of trefoil peptides, occurs through binding to a specific receptor is still unresolved. Saturable porcine SP binding activity within iso- lated rat intestinal membranes, with 2 affinity states, has been described (46, 47). Subsequently, an ITF-derived fusion protein and ${ }^{125}$ I-labeled rat ITF was shown to bind gut tissue sections in vitro, with strong signals in the gastric neck region and Paneth's cells (48, 49). A trefoil peptide-binding protein of approximately $28 \mathrm{kDa}$ has been detected after cross-linking studies (50). At this time, however, these entities are incompletely characterized, and molecular cloning of these proteins has not been reported. We have found in this study that ITF does not appear to directly bind the EGF-R or colocalize with the EGF-R under nonpermissive conditions but does colocalize with the transferrin receptor to clathrin-coated pits. Trefoil-mediated activation of the EGF-R, therefore, may occur indirectly, possibly by recruitment of adaptor molecules or Ras. The trefoil peptides may also activate after endocytosis. These findings do not necessarily invoke a specific receptor, but suggest an alternative hypothesis where trefoil-mediated events occur following internalization of the peptides.

We have shown in this study that the increase in SP and pS2 mRNA following trefoil peptide stimulation is most likely a transcriptional response. SP and $p S 2$ gene promoters were activated 5 - to 10 -fold in response to ITF. The cis-acting elements mediating this transcriptional enhancement were not identified

\section{Figure 6}

EGF-R-mediated stimulation of pS2 transcription. (a) Phosphorylation of EGF$R$ in AGS cells by ITF. Confluent, serum-starved AGS cells were stimulated with ITF $(10 \mathrm{ng} / \mu \mathrm{L})$ for the indicated times in minutes or with EGF for 5 minutes before cell lysis. Proteins were immunoprecipitated with anti-EGF-R, separated on SDS-PAGE, transferred to PVDF membranes, and blotted with the monoclonal anti-phosphotyrosine antibodies 4G10/PY20. (b) Transcriptional regulation of $\mathrm{pS} 2$ by trefoil peptides is mediated by EGF-R. AGS cells were transfected with the pS2-luciferase reporter construct in the presence or absence of a dominant-negative EGF-R (pHER653) expression construct, then stimulated 12 hours later with EGF or ITF. Relative light units (RLU) corrected for transfection efficiency were assessed by cotransfection with $1 \mu \mathrm{g}$ pRL-TK (Renilla) luciferase.

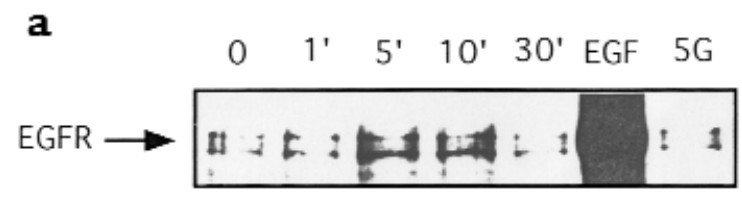

b

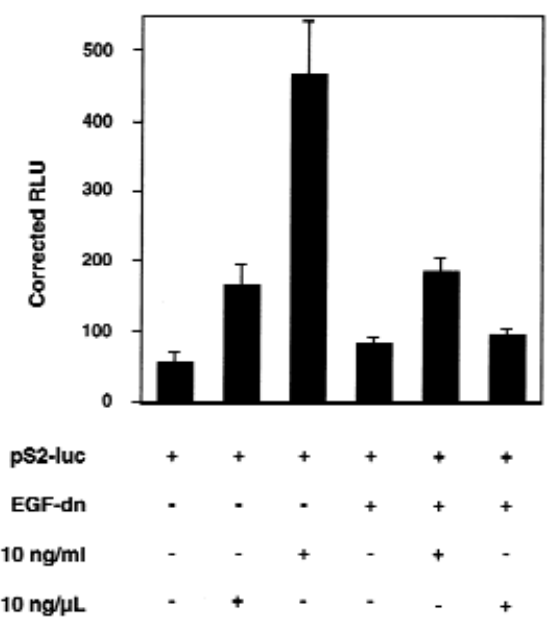



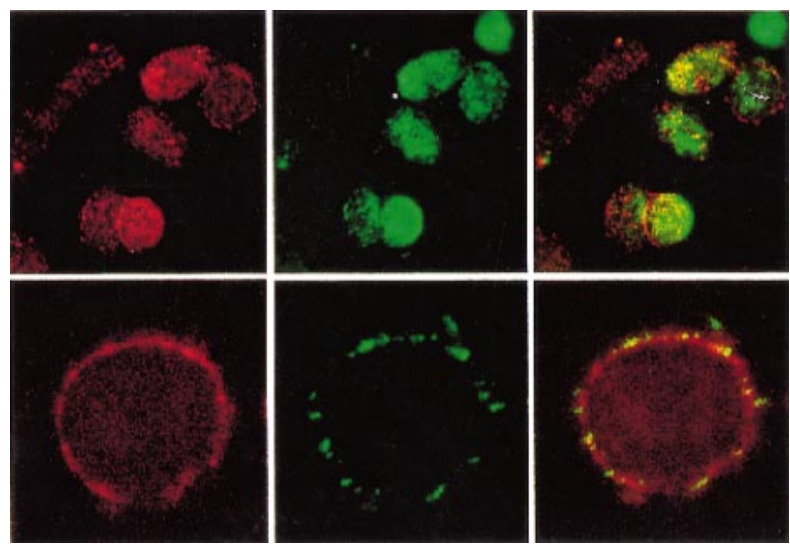

\section{Figure 7}

ITF binding to cells under nonpermissive conditions colocalizes with the transferrin receptor, but not with the EGF-R. AGS cells (pictured) or HT29 cells were grown on glass, cooled to $4^{\circ} \mathrm{C}$, and an excess $(10 \mu \mathrm{g} / \mathrm{mL})$ of ITF or ITF-thioredoxin was added. Incubations with primary antibodies were as follows. Top left: monoclonal anti-thioredoxin; top center: rabbit anti-EGF-R; top right: merged image showing minimal colocalization of ITF (red) and EGF-R (green). Similar staining was seen with anti-ITF staining in combination with monoclonal anti-EGF-R. Original magnification: $\times 200$. Bottom left: rabbit anti-ITF; bottom center: clathrin-coated pits seen with monoclonal antibody to the transferrin receptor $C D 71$; bottom right: merged image showing predominantly colocalization of ITF staining with CD-71. Original magnification: $\times 400$. in this study but were present within the 820-bp and 1,100-bp SP and pS2 promoters, respectively. Previously, an enhancer element responsive to signals initiated by Ras, EGF, and phorbol ester was identified in the human $p S 2$ promoter, and this enhancer activity was allocated to the imperfect palindrome GGTCACGGTGGCC, present $450 \mathrm{bp}$ upstream of the initiator ATG (26). This sequence is not found in the human SP promoter described here or in the ITF promoter sequences reported to date; nor do the $S P / p S 2$ promoters contain canonical MAPK response elements such as AP-1, SRE, or CRE motifs. Therefore, identification of the enhancer elements necessary for trefoil interregulation will be a focus of further studies.

The rise in steady-state trefoil mRNA after stimulation was augmented by cycloheximide; this feature, together with the rapidity of stimulation, suggests that the trefoils may act as immediate-early genes. Regulation of gastric trefoil expression by EGF-R ligands, some of which are also immediate-early genes capable of cross-induction (35), has been proposed $(23,36)$; indeed, pS2 transcription is markedly induced by EGF in the breast cancer cell line MCF7 (26). In the present study, using cells of gastrointestinal tract origin, EGF was a modest stimulant of trefoil expression (Figure 2) at the concentration used. Instead, trefoil peptides themselves appear to act via EGF-R to initiate a signal transduction cascade terminating in trefoil gene induction. The finding that the EGFR-trefoil relationship mediates transcriptional responses to ITF provides insight into otherwise paradoxical observations. In healing gut mucosa, induction of EGF-R has been described $(41,42)$, but no single EGF-R ligand has been demonstrated to be critical for gastrointestinal healing. Thus, TGF- $\alpha$ null mice appear to have normal healing after induction of gastric ulcers (36). In contrast, the colonic erosions induced by oral dextran sodium sulfate, while promptly repaired in wild-type mice, are fulminant in ITF null mice, leading to death of the animal. This defect can be reversed by rescue with topical ITF (11).

How does the gastric mucous neck cell, the site of SP gene expression, "see" surface-expressed pS2, or more problematic, ITF, a product of the intestinal goblet cell? Though the dynamics of gastric mucus flow are essentially unknown, it is possible that pS2 generated and secreted by the surface mucous cells may be swept proximally to the gland neck. Although ITF is expressed and secreted in the base of gastric glands, peptide levels are only approximately $1 \%$ those found in the intestine (16). However, it is possible that this level may be sufficient to sustain SP induction. Alternatively, substantial ITF is expressed in the duodenum and may bathe the gastric antrum as a component of duodenogastric refluxate. Circulating trefoil peptides may also be responsible for this crossregulation. SP and PS2 expression by endocrine cells of the gut has been reported (23), and systemic administration of SP was able to protect rats from gastric damage caused by indomethacin (13). Consistent with this possibility is the observation of increased ITF expression in uninjured gastric mucosa lying opposite injured and regenerating gastric mucosa (Taupin, D.R., et al., unpublished observations).

A further possibility is that the degree of trefoil expression is programmed in pluripotent cells in the proliferative zone of the gastric gland. In this context, autocrine stimulation of SP expression might be partly dependent on the expression of ITF (or pS2) by that cell, dictating subsequent expression by differentiated progeny. Thus, cells of relatively undifferentiated morphology in regenerating gastric glands are capable of expressing the full trefoil repertoire (21). Detailed characterization of epithelial stem cells present in different regions of the gastrointestinal tract may provide further insight.

In aggregate, these data provide a paradigm for the rapid self-sustaining induction of trefoil transcription after mucosal injury via EGF-R activation and through the Ras/MEK/MAP kinase signaling pathway, leading to activation of trefoil genes through cis-acting regulatory regions. A role for trefoil-induced signals in the maintenance of metaplastic gut lineages also appears likely. As MAP kinase activation appears to be necessary for cell migration (37), effects of trefoil peptides on cell restitution may also occur through this pathway. Nevertheless, the possibility of trefoil peptide internalization suggests that coupling to alternate signaling pathways is also possible. 


\section{Acknowledgments}

The authors are grateful to Dennis Brown for helpful suggestions and Phil Davis for technical assistance. This work was supported by grants from the Crohn's and Colitis Foundation of America and grants from the National Institutes of Health (DK-41557 and DK-43351).

1. Nusrat, A., Delp, C., and Madara, J.L. 1992. Intestinal epithelial restitution: Characterization of a cell culture model and mapping of cytoskeletal elements in migrating cells. J. Clin. Invest. 89:1501-1511.

2. Dieckgraefe, B.K., Stenson, W.F., and Alpers, D.H. 1996. Gastrointestinal epithelial response to injury. Curr. Opin. Gastroenterol. 12:109-114.

3. Santos, M.F., et al. 1997. Rho proteins play a critical role in cell migration during the early phase of mucosal restitution. J. Clin. Invest. 100:216-225.

4. Ciacci, C., Lind, S.E., and Podolsky, D.K. 1993. Transforming growth factor beta regulation of migration in wounded rat intestinal epithelial monolayers. Gastroenterology. 105:93-101.

5. Dignass, A.U., Tsunekawa, S., and Podolsky, D.K. 1994. Fibroblast growth factors modulate intestinal epithelial cell growth and migration. Gastroenterology. 106:1254-1262.

6. Nusrat, A., et al. 1994. Hepatocyte growth factor/scatter factor effects on epithelial: Regulation of intercellular junctions in transformed and nontransformed cell lines, basolateral polarization of c-met receptor in transformed and natural intestinal epithelia, and induction of rapid wound repair in a transformed model epithelium. J. Clin. Invest. 93:2056-2065.

7. Dignass, A.U., Lynch-Devaney, K., and Podolsky, D.K. 1994. Hepatocyte growth factor/scatter factor modulate intestinal epithelial cell proliferation and migration. Biochem. Biophys. Res. Comm. 202:701-709.

8. Dignass, A.U., and Podolsky, D.K. 1993. Cytokine modulation of intestinal epithelial cell restitution: Central role of transforming growth factorB. Gastroenterology. 105:1323-1332.

9. Dignass, A., Lynch-Devaney, K., Hindon, H., Thim, L., and Podolsky, D.K. 1994. Trefoil peptides promote epithelial restitution through a TGF- $\beta$ independent pathway. J. Clin. Invest. 94:376-383.

10. Sands, B.E., and Podolsky, D.K. 1996. The trefoil peptide family. Annu. Rev. Physiol. 58:253-273.

11. Mashimo, H., Wu, D.-C., Podolsky, D.K., and Fishman, M.C. 1996. Impaired defense of intestinal mucosa in mice lacking intestinal trefoil factor. Science. 274:262-265.

12. Babyatsky, M.W., deBeaumont, M., Thim, L., and Podolsky, D.K. 1996. Oral trefoil peptides protect against ethanol- and indomethacin-induced gastric injury in rats. Gastroenterology. 110:489-497.

13. Playford, R.J., et al. 1995. Human spasmolytic polypeptide is a cytoprotective agent that stimulates cell migration. Gastroenterology. 108:108-116

14. Lefebvre, O., et al. 1996. Gastric mucosa abnormalities and tumorigenesis in mice lacking the pS2 trefoil protein. Science. 274:259-262.

15. Jeffrey, G.P., et al. 1994. Spasmolytic polypeptide: a trefoil peptide secreted by rat gastric mucous cells. Gastroenterology. 106:336-345.

16. Taupin, D.R., Pang, K.C., Green S.P., and Giraud, A.S. 1995. The trefoil peptides spasmolytic polypeptide and intestinal trefoil factor are major secretory products of the rat gut. Peptides. 16:1001-1005

17. Lefebvre, O., et al. 1993. The mouse one Pdomain (pS2) and two P-domain (raSP) genes exhibit distinct patterns of expression. J. Cell. Biol. 122:191-198.

18. Itoh, H., et al. 1996. cDNA cloning of rat pS2 peptide and expression of trefoil peptides in acetic acid-induced colitis. Biochem. J. 318:939-944.

19. Suemori, S., Lynch-Devaney, K., and Podolsky, D.K. 1991. Identification and characterization of rat intestinal trefoil factor: tissue and cell-specific member of the trefoil protein family. Proc. Natl. Acad. Sci. USA. 88:11017-11021.

20. Podolsky, D.K., et al. 1993. Identification of human intestinal trefoil factor: goblet cell-specific expression of a peptide targeted for apical secretion. J. Biol. Chem. 268:6694-6702.

21. Alison, M.R., et al. 1995. Experimental ulceration leads to sequential expression of spasmolytic polypeptide, intestinal trefoil factor, epidermal growth factor and transforming growth factor alpha mRNAs in rat stomach. J. Pathology. 175:405-414.

22. Rio, M., et al. 1991. Induction of pS2 and hSP genes as markers of mucosal ulceration of the digestive tract. Gastroenterology. 100:375-379.

23. Wright, N.A., et al. 1993. Trefoil peptide gene expression in gastrointestinal epithelial cells in inflammatory bowel disease. Gastroenterology. 104:12-20.

24. Hanby, A.M., et al. The expression of the trefoil peptides $\mathrm{pS} 2$ and human spasmolytic polypeptide (hSP) in gastric metaplasia of the proximal duodenum: implications for the nature of 'gastric metaplasia.' J. Pathol. 169:355-360.

25. Sambrook, J., Fritsch, E., and Maniatis, T. 1989. Molecular cloning: a laboratory manual. 2 nd edition. Cold Spring Harbor Laboratory Press. Cold Spring Harbor, NY. 2.69-2.72.

26. Nunez, A.-M., Berry, M., Imler, J.L., and Chambon, P. 1989. The $5^{\prime}$ flanking region of the pS2 gene contains a complex enhancer region responsive to oestrogens, epidermal growth factor, a tumour promoter (TPA), the c-Ha-ras oncoprotein and the c-jun protein. EMBO J. 8:823-829.

27. Ward, Y., et al. 1994. Control of MAP kinase activation by the mitogen-induced threonine/tyrosine phosphatase PAC1. Nature. 367:651-654.

28. Feig, L.A., and Cooper, G.M. 1988. Inhibition of NIH 3T3 proliferation by a mutant ras protein with preferential affinity for GDP. Mol. Cell. Biol. 8:3235-3243.

29. Wagner, M., et al. 1996. Expression of a truncated EGF receptor is associated with inhibition of pancreatic cell growth and enhanced sensitivity to cisplatinum. Int. J. Cancer. 68:782-787.

30. Chung, D.C., Brand, S.J., and Tillotson, L.G. 1995. Mutually exclusive interactions between factors binding to adjacent Sp1 and AT-rich elements regulate gastrin gene transcription in insulinoma cells. J. Biol. Chem. 270:8829-8836.

31. Kanai, M., Mullen, C., and Podolsky, D.K. 1998. Intestinal trefoil factor induces inactivation of extracellular signal-related protein kinase in intestinal epithelial cells. Proc. Natl. Acad. Sci. USA. 95:178-182.

32. Ogata, H., Inoue, N., and Podolsky, D.K. 1998 Identification of a goblet cell-specific enhancer element in the rat intestinal trefoil factor gene promoter bound by a goblet cell nuclear protein. J. Biol. Chem. 273:3060-3067.

33. Gött, P., et al. 1996. Human trefoil peptides: genomic structure in $21 \mathrm{q} 22.3$ and coordinated expression. Eur. J. Hum. Genet. 4:308-315.
34. Chinery, R., Poulsom, R., and Cox, H.M. 1996 The gene encoding mouse intestinal trefoil factor: Structural organization, partial sequence analysis and mapping to murine chromosome 17q. Gene. 171:249-253.

35. Barnard, J.A., et al. 1994. Auto- and cross-induction within the mammalian epidermal growth factor-related peptide family. J. Biol. Chem 269:22817-22822.

36. Cook, G.A., Yeomans, N.D., and Giraud, A.S. 1997. Temporal expression of trefoil peptides in the TGF- $\alpha$ knockout mouse after gastric ulceration. Am. J. Physiol. 272:G1540-G1549.

37. Klemke, R.L., et al. 1997. Regulation of cell motility by mitogen-activated protein kinase. J. Biol. Chem. 137:481-492.

38. Davis, R.J. 1993. The mitogen-activated protein kinase signal transduction pathway. J. Biol. Chem. 268:14553-14556.

39. Alessi, D.R., Cuenda, A., Cohen, P., Dudley, D.T., and Saltiel, A.R. 1995. PD098059 is a specific inhibitor of the activation of mitogen-activated protein kinase in vitro and in vivo. J. Biol. Chem. 270:27489-27494.

40. Rio, M.C., et al. 1988. Breast cancer-associated pS2 protein: synthesis and secretion by normal stomach mucosa. Science. 241:705-708.

41. Hansson, H.-A., Hong, L., and Helander, H.F. 1990. Changes in gastric EGF, EGF receptors and acidity during healing of gastric ulcer in the rat. Acta Physiol. Scand. 128:241-242.

42. Tarnawski, A., Stachura, J., Durbin, T., Sarfeh, I.J., and Gergely, H. 1992. Increased expression of epidermal growth factor receptor during gastric ulcer healing in rats. Gastroenterology. 102:695-698.

43. Liu, D., et al. 1997. Phosphorylation of $\beta$-catenin and epidermal growth factor receptor by intestinal growth factor. Lab. Invest. 77:557-563.

44. Thim, L. 1989. A new family of growth factor-like peptides. 'Trefoil' disulphide loop structures as a common feature in breast cancer associated peptide (pS2), pancreatic spasmolytic polypeptide (PSP), and frog skin peptides (spasmolysins). FEBS Lett. 250:85-90.

45. Vieira, A.V., Lamaze, C., and Schmid, S.L. 1996. Control of EGF receptor signaling by clathrinmediated endocytosis. Science. 274:2086-2089.

46. Frandsen, E.K., Jørgensen, K.H., and Thim, L. 1986. Receptor binding of pancreatic spasmolytic polypeptide (PSP) in rat intestinal mucosal cell membranes inhibits the adenylate cyclase activity. Regul. Pept. 16:291-297.

47. Frandsen, E.K. 1988. Receptor binding of pancreatic spasmolytic polypeptide in intestinal mucosal cells and membranes. Regul. Pept. 20:45-52

48. Rasmussen, T.N., Thim, L., Raaberg, L. Hurling, H., and Holst, J.J. 1990. Pancreatic spasmolytic polypeptide, a potential growth factor for the intestine: localisation and control of secretion. Digestion. 46(Suppl. 2):90.

49. Chinery, R., Poulsom, R., Elia, G., Hanby, A.M., and Wright, N.A. 1993. Expression and purification of a trefoil peptide motif in a $\beta$-galactosidase fusion protein and its use to search for trefoil binding sites. FEBS Lett. 212:557-563.

50. Chinery, R., and Cox, H.M. 1995. Immunoprecipitation and characterization of a binding protein specific for the peptide, intestinal trefoil fac tor. Peptides. 16:749-755.

51. Mashimo, H., Podolsky, D.K., and Fishman, M.C. 1995. Structure and expression of murine intestinal trefoil factor: High evolutionary conservation and postnatal expression. Biochem. Biophys. Res. Comm. 210:31-37. 